\title{
INVESTIGACIONES
}

\section{Ejercicio de la autoridad en profesores de educación secundaria de Chile*}

\author{
How do teachers exercise authority in secondary schools in Chile? \\ Marisa Meza Pardo, ${ }^{a}$ Guillermo Zamora Poblete, ${ }^{a b}$ Pilar Cox Vial ${ }^{a c}$ \\ ${ }^{a}$ Facultad de Educación, Pontificia Universidad Católica de Chile \\ Telf.: (56) 23545385. Correo electrónico: mmeza@uc.cl \\ 'Telf.: (56) 23545373. Correo electrónico: gzamora@puc.cl \\ 'Telf.: (56) 23545342. Correo electrónico: pcox@uc.cl
}

\begin{abstract}
El presente estudio se propone caracterizar las formas en que profesores y profesoras ejercen autoridad, y examinar la relación entre estas formas y el grupo socioeconómico (GSE) de los estudiantes. Se examinan las interacciones con estudiantes de 18 profesores de educación secundaria que han sido reconocidos previamente como los docentes con mayor autoridad según sus alumnos. El estudio utiliza técnicas cualitativas y estadísticos descriptivos. Se analizan las solicitudes o peticiones que plantea el profesor y las estrategias que utiliza para lograr que sus estudiantes las acepten. Los resultados indican la existencia de tres formas de ejercicio de autoridad pedagógica: la 'autoridad explícita', la 'autoridad compartida' y la 'autoridad como misión moral'. La primera y la tercera forma se presentan especialmente en el GSE Bajo. La segunda, en el GSE Medio y Alto.
\end{abstract}

Palabras clave: autoridad pedagógica, poder del profesor, interacción profesor y estudiantes, Educación secundaria.

\section{ABSTRACT}

The objective of this study is to characterize the ways in which teachers exercise authority, and to examine the relationship between types of authority and students' socioeconomic status (SES). Classes from 18 secondary teachers, who had been previously recognized by their students as exhibiting more authority than other teachers, were observed. Requests from the teachers, their topics, and the strategies used to accomplish students accepting the request were analyzed; the study uses qualitative techniques and statistical descriptions. Results show the existence of three types of pedagogical authority: 'Explicit authority', highly directive and focused on academic contents; 'shared authority', in which the teacher shares power with the students, focused as well in academic contents; and 'moral authority', highly directive and focused on the moral guidance of students. The first and the third one are especially manifested in low SES, while the second is often seen more in middle and low SES.

Key words: pedagogical authority, teacher-student interaction, secondary education, learning environment.

\footnotetext{
El presente estudio forma parte de la investigación "Ensayos y tentativas en la construcción de la autoridad pedagógica en profesores principiantes de enseñanza media: significados, procesos, dificultades y logros durante los primeros años de ejercicio profesional", financiado por la Comisión Nacional de Investigación Científica y Tecnológica de Chile (CONICYT) a través del proyecto FONDECYT No 1160625.
} 


\section{PROBLEMAS Y OBJETIVOS}

Diversos estudios han planteado las tensiones que actualmente presenta el ejercicio de la autoridad pedagógica en la educación secundaria. Estudios latinoamericanos han hecho referencia a los fenómenos de violencia escolar, los cuales se caracterizan generalmente por agresiones entre los estudiantes y también entre docentes y alumnos (Tedesco \& Tenti, 2002; Zamora, Meza \& Cox, 2015; Zerón, 2006). En Chile, esta situación ha sido constatada en diversos estudios. La "IX Encuesta Nacional a los Actores del Sistema Educativo Chileno" (Centro de Investigación y Desarrollo de la Educación, 2012) señala que la indisciplina es uno de los problemas más recurrentes en el aula según los profesores (31,3\% de los docentes así lo indica). A su vez, el 18,8\% de los profesores señala que es frecuente un trato verbal o gestual irrespetuoso hacia el docente o directivo, situación más fuerte en los colegios públicos $(29,2 \%$ de los profesores lo reporta). Ruffinelli (2014) constata que la principal dificultad en el ejercicio docente corresponde a problemas de conducta de los estudiantes (señalado por 33,2\% de los profesores principiantes). Esta situación es más fuerte cuando se trabaja con estudiantes de grupo socioeconómico (GSE) Bajo, señalado por el 33,4\% de los profesores. Ello también es expresado por los propios estudiantes secundarios; de acuerdo al informe PISA 2009, Chile constituye el sexto país (entre 66 examinados) donde los estudiantes perciben más desorden en clases sin que el profesor logre intervenir con éxito.

En este contexto, cabe preguntarse, ¿cómo ejercen autoridad los profesores que son obedecidos por sus estudiantes? ¿Existen diferencias en el ejercicio de la autoridad según Grupo Socioeconómico (GSE) de los alumnos?

Los objetivos del presente estudio son:

- Caracterizar las formas en que los profesores ejercen autoridad en las interacciones en el aula.

- Comparar las formas de ejercicio de la autoridad en relación al GSE de los estudiantes a los que enseña el profesor.

\section{ANTECEDENTES}

\subsection{AUTORIDAD DEL PROFESOR}

Adoptando la perspectiva social de Weber (1983), se entiende por autoridad un tipo de poder en el cual los individuos gobernados deciden obedecer a otro (aceptar sus demandas) debido a que otorgan legitimidad a sus mandatos. La autoridad surge en el reconocimiento, el cual según Weber (1983) puede estar motivado por la tradición, el carisma o la legalidad del ordenamiento establecido. La perspectiva social pone énfasis en el carácter relacional de la autoridad: no hay autoridad en soledad, siempre se requiere de un otro que la reconozca. Por lo tanto, la autoridad pedagógica se entiende como la relación asimétrica que establece el profesor con los alumnos que le permite influir sobre ellos y conseguir que acepten sus demandas y orientaciones en el ámbito de la enseñanza y aprendizaje (Bourdieu \& Passeron, 1996).

En la sociedad tradicional (siguiendo las categorías de Weber, 1983) la construcción de la autoridad no constituyó un problema tan apremiante como hoy pues esta era garantizada 
por fuertes instituciones sociales y el peso de la tradición y la religión (Arendt, 1996; Dubet, 2006). El profesor era respetado y obedecido en cuanto encarnaba una moral superior. Siguiendo a Dukheim (2009), el maestro se asemejaba a un sacerdote laico, pues interpretaba las grandes ideas morales de su tiempo y de su país. Lo mismo que el sacerdote era intérprete de su dios; el maestro era intérprete de la moral de su sociedad.

Con el advenimiento de la modernidad, se produce un desencantamiento de los fundamentos de la autoridad y el declive de las instituciones tal como se las había conocido. En esta línea, Martuccelli (2009) señala que actualmente la autoridad constituye un problema general, pues el ingreso a una sociedad secularizada y democrática implica la sospecha del orden social heredado y del sustento de la autoridad en valores religiosos. Dubet y Martuccelli (1998) agregan que la escuela de hoy está lejos de presentarse como una máquina que forma individuos según un esquema social común y legitimado por todos. A nivel latinoamericano, Batallán (2003) lo ha planteado de este modo: "el viejo sistema vertical, que se inicia con el mando de los directivos sobre los maestros y continúa con el de estos sobre los niños, ha entrado en crisis, sin que haya sido reemplazado por alternativas ni pedagógicas, ni políticas" (p. 684). En los adolescentes, el respeto y la confianza hacia el maestro no son dados como algo natural e indiscutido. Los profesores no tienen garantizada la obediencia, la escucha y el reconocimiento. De aquí que la pregunta que tensiona permanentemente a los profesores es: ¿cómo construir una relación de autoridad pedagógica con los estudiantes en medio de sociedades cada vez menos jerárquicas y más sospechosas de las asimetrías, con instituciones escolares en declive?

\subsection{EJERCICIO DE AUTORIDAD}

Escasos son los estudios que examinan el ejercicio de la autoridad en la sala de clases en la educación actual. Entre ellos destaca el examen de Bourdieu y Passeron (1996) que da cuenta de que la autoridad pedagógica tiene la función de legitimar la cultura que arbitrariamente selecciona la institución escolar. La legitimación de la cultura es predominantemente simbólica y se ejerce en la relación comunicativa. Bourdieu y Passeron (1996) advierten que esta comunicación no es exclusivamente un proceso interpersonal. La autoridad del profesor descansa tanto en lo que valora la institución escolar, como en el hecho de que el mensaje que dirige es socialmente apreciado por los estudiantes.

Bernstein (2001) observa que los profesores y estudiantes interactúan en la sala de clases negociando el modo de transmisión y adquisición de los contenidos escolares. Esta negociación tiene límites: las imposiciones del sistema educativo nacional, especialmente el currículum escolar. Dentro de estos límites, el profesor ejerce el control ('enmarcamiento' en palabras de Bernstein, 2001). De este modo, la autoridad del profesor está sostenida tanto por el poder que el currículum le proporciona a la asignatura que imparte, como también por la forma de control o enmarcamiento que establece con los estudiantes. Bersntein (1989) observó que el control se puede desplegar desde formas posicionales a formas personalizadas. El modo posicional está vinculado a normas comunes previamente establecidas a las que deben ajustarse los estudiantes debido a su rol. Por ejemplo: "como alumno debes estar callado para entender la materia". En cambio, en el modo personalizado la norma no es previa, ni exigida directamente; más bien es el alumno quien la alcanza u opta por ella. Por ejemplo, "te haría bien realizar esta tarea pues te ayudará a tu desarrollo personal”. Bernstein (1989) observa una relación entre clase social y modos 
de control. En la clase social más baja se tiende a ejercer un control posicional; mientras que en clase media se utiliza uno más personal. De ello se desprende un desafío para los profesores: ¿Cómo ejercer el control en la sala de clases de tal modo que haga sentido a los alumnos?

En la línea de tipos de control y clase social, es relevante considerar el trabajo de Delpit (1995). La autora identifica que en las salas de clases existen reglas de poder que pueden ser más o menos explícitas. En general, las reglas de poder son un reflejo de la cultura de los que tienen poder. Para los estudiantes de sectores medios y altos, que conocen esta cultura, no es tan valorada la explicitación de las reglas para obedecer. En cambio, en los estudiantes de sectores desaventajados, distantes del poder, se presenta una suerte de disonancia cultural cuando las reglas son implícitas, pues no tienen el código para comprender qué se les solicita. Por ello, los estudiantes de sectores desaventajados valoran más cuando la pedagogía es explícita y las peticiones del profesor son directas. Delpit (1995) agrega que la autoridad del profesor con estudiantes del grupo social bajo, requiere que se movilice con mayor fuerza: los profesores deben exhibir su poder personal permanentemente, establecer un sistema de reconocimiento y de motivación de los estudiantes que los incorpore en forma continua y capture su atención constantemente. En contraste, el profesor que enseña a grupos sociales medio y alto no necesita explicitar su poder: la autoridad ya está envestida en el rol de ser maestro.

Es importante advertir que hoy se sigue discutiendo la relevancia del control como fundamento de la autoridad. Dubet (2006) observa que actualmente los estudiantes "ya no están dispuestos a reconocer la autoridad del profesor como natural y como obvia; esperan ser convencidos de la utilidad de los estudios, ya sea de su interés intelectual o de su interés social" (Dubet, 2006, p. 160). La obediencia ciega ha sido reemplazada por el principio de reciprocidad: se respeta a los profesores que son respetuosos con ellos. El respeto mutuo es, a su vez, combinado con una valoración diferencial de las asignaturas: la percepción de rentabilidad es variable y se construye mediante decisiones estratégicas y en la relación personal que se tiene con el docente. Asimismo, Dubet (2006) observa que actualmente los estudiantes presentan una poliarquía de valores, esperan que se les reconozca su diversidad y buscan que sus profesores les den espacio para manifestar su individualidad. Dicho de otro modo, actualmente ya no se espera que la escuela discipline a los alumnos para convertirlos en las personas que la institución escolar estima moralmente correcta, sino que se espera que los profesores ayuden a expandir una personalidad única.

El estudio de Harjünen (2009) en las escuelas secundarias finlandesas también da cuenta de que el reconocimiento de la autoridad tiene importantes componentes referidos al reconocimiento personal que experimentan los estudiantes. La investigadora constata tres dimensiones que indican los estudiantes para reconocer como autoridad a un profesor. La primera dimensión es de tipo relacional e incluye aspectos como sentirse escuchado por el maestro y con espacios de autonomía en las clases: los estudiantes valoran especialmente al profesor que se relaciona con ellos con respeto y les reconoce sus particularidades. La segunda dimensión es didáctica y se refiere al apoyo y motivación hacia el aprendizaje que esperan recibir los estudiantes de sus profesores. La tercera se relaciona con las normas y la ética: los estudiantes esperan que sus profesores sean modelos en valores y quieren sentirse seguros y valorados en sus clases. 


\section{METODOLOGÍA}

El presente estudio adopta un diseño exploratorio, y utiliza técnicas cualitativas y estadísticos descriptivos no paramétricos.

\subsection{SELECCIÓN DE PARTICIPANTES}

Se observan las clases de 18 de profesores en el nivel de II año de educación secundaria (con estudiantes de 15 a 16 años), de establecimientos escolares de la ciudad de Santiago de Chile. Los docentes han sido seleccionados a partir de un estudio previo (Zamora et al., 2015) que consultó a 936 estudiantes quién es el profesor que representa mayor autoridad. Los 18 profesores seleccionados son aquellos que presentan mayor porcentaje de menciones por parte de los estudiantes.

Se clasifican a los 18 profesores considerando el GSE de los estudiantes a los que enseñan. Para clasificar el GSE se utiliza las categorías socioeconómicas del establecimiento proporcionada por el Ministerio de Educación de Chile. Estas categorías están basadas en el promedio de escolaridad de los padres de los estudiantes y el ingreso económico mensual del hogar (ver Tabla 1).

Tabla 1. Características GSE del establecimiento y los estudiantes

\begin{tabular}{|c|c|c|c|}
\hline \multirow{2}{*}{$\begin{array}{l}\text { Grupo Socioeconómico } \\
\text { del establecimiento }\end{array}$} & \multicolumn{2}{|l|}{ Promedio Años de estudio } & \multirow{2}{*}{ Promedio Ingreso Hogar mensual U\$ } \\
\cline { 2 - 4 } & Madre & Padre & \\
\hline Alto & Más de 14 & Más de 15 & Más de U\$ 2.400 \\
\hline Medio & $12-13$ & $12-13$ & Entre U\$ 650-1.100 \\
\hline Bajo & Hasta 9 & Hasta 9 & Menos de U\$ 430 \\
\hline
\end{tabular}

Fuente: elaboración propia.

De los 18 profesores observados, 6 de ellos enseñan a GSE Alto, 6 a Medio y 6 a Bajo. Existe una equivalencia de género (9 mujeres y 9 varones). En promedio, los docentes tienen 22,38 años de servicio. Sus principales características se resumen en la Tabla 2. 
Tabla 2. Caracterización de profesores seleccionados

\begin{tabular}{|c|c|c|c|c|c|}
\hline \multirow{2}{*}{$\begin{array}{l}\text { GSE de la } \\
\text { escuela }\end{array}$} & \multirow{2}{*}{$\begin{array}{c}\mathrm{N}^{\circ} \text { profesores } \\
\text { observados }\end{array}$} & \multicolumn{2}{|c|}{ Género } & \multirow{2}{*}{$\begin{array}{l}\text { Años de } \\
\text { servicio }\end{array}$} & \multirow{2}{*}{$\begin{array}{l}\text { Asignaturas } \\
\text { que imparten }\end{array}$} \\
\hline & & Femenino & Masculino & & \\
\hline Alto & 6 & 1 & 5 & 28,6 & $\begin{array}{l}\text { Química: 2; Historia: } 1 \\
\text { Matemática: 2; Física:1 }\end{array}$ \\
\hline Medio & 6 & 5 & 1 & 13,5 & $\begin{array}{l}\text { Biología:2; Matemática:1; Artes } \\
\text { Visuales: } 1 \text {; Lenguaje: 1; Física:1 }\end{array}$ \\
\hline Bajo & 6 & 3 & 3 & 14,1 & $\begin{array}{l}\text { Historia: 4; Religión:1 } \\
\text { Lenguaje:1; Química:1 }\end{array}$ \\
\hline TOTAL & 18 & 9 & 9 & 22,38 & $\begin{array}{l}\text { Historia: 5; Matemática: } 3 \\
\text { Química: 3; Física: } 2 \\
\text { Biología:2; Lenguaje: } 2 \\
\text { Artes Visuales: } 1 \text {; Religión:1 }\end{array}$ \\
\hline
\end{tabular}

Fuente: elaboración propia.

\subsection{OBSERVACIÓN DE CLASES}

Se observan tres clases de cada profesor durante los primeros meses del año escolar 2014. El registro de las interacciones se realiza con la 'Pauta de Observaciones de Autoridad Pedagógica' (Meza, Cox \& Zamora, 2015). La pauta recoge información de interacciones lingüísticas entre profesor y alumnos, focalizada en episodios en que se desarrollan solicitudes o peticiones entre los actores. De cada petición, se registran las siguientes dimensiones: a) Persona que formula la petición (profesor o alumno), b) Contenidos de las peticiones (académico o de convivencia), c) Estrategias del profesor para aumentar la probabilidad de que la petición sea aceptada por los alumnos. Se define por estrategia cualquier medio o acción que acompaña a la petición con la perspectiva de que el alumno la acepte. Además, se toman notas de campo -registro amplio- del comportamiento lingüístico y no lingüístico del profesor.

\subsection{ANÁLISIS DE LA INFORMACIÓN}

Para establecer las formas de ejercicio de autoridad se realiza el siguiente proceso de análisis:

Fase 1. De las tres clases observadas a cada profesor, se establece un promedio en la dimensión "a" y de la "b". Para la dimensión "a" se establece el porcentaje promedio de las tres clases en que las peticiones son formuladas por el propio profesor. Para la dimensión "b", se establece el porcentaje promedio de las tres clases en que las peticiones se refieren al ámbito académico.

Fase 2. Se agrupan a los profesores que presentan similar promedio en la dimensión "a" $y$ " $b$ ". Se establece como criterio de agrupamiento que el puntaje entre ellos no sobrepase, en estas dimensiones, los 10 puntos porcentuales.

Fase 3. Se identifican las estrategias -dimensión "c"- que coinciden al interior de cada grupo de profesores. 
Fase 4. Se identifican rasgos similares en el comportamiento lingüístico y no lingüístico de los profesores de cada grupo.

Fase 5. Para explorar la asociación, no paramétrica, entre las formas de ejercicio de autoridad y el GSE del establecimiento en que se desempeñan los profesores observados, se analizan frecuencias y medias.

\section{RESULTADOS}

A continuación, se presentan los resultados del estudio considerando las diversas dimensiones examinadas y los objetivos de la investigación.

\subsection{FORMULACIÓN DE PETICIONES}

Son los profesores quienes principalmente formulan las peticiones en las clases: $82,2 \%$ de ellas las hace el profesor y el 17,8\% los alumnos. Existen importantes diferencias según el GSE de los estudiantes. En el GSE Bajo, la formulación de peticiones por parte del profesor se presenta en un 91,4\%; en el Medio, en un 86,6\% y en el Alto, en un 67,0\%.

\subsection{CONTENIDOS DE LAS PETICIONES}

La mayoría de las peticiones son de tipo 'académico' (78,9 \% de ellas). Estas peticiones se refieren a temas específicamente vinculados con el contenido instruccional: pedir una respuesta frente a la materia vista en clases, pedir una aclaración sobre un tema o pedir que se realice un informe o investigación. Las peticiones 'no académicas' se refieren a regulaciones de las conductas sociales no directamente relacionadas con la materia de la clase. Incluyen peticiones de ordenar la sala, sentarse, estar en silencio, asistir a un compañero o no agredirlo. Las peticiones 'no académicas' son significativamente menos frecuentes: $21,1 \%$ de las peticiones.

Existe una débil relación entre el porcentaje de contenido académico de la petición y el GSE del establecimiento al que pertenece el profesor. En el GSE Bajo se presenta el contenido académico en el 75,0\% de las peticiones; en el Medio, en el 77,6\% y en el Alto, en el $84,8 \%$.

\subsection{FORMAS DE EJERCICIO DE LA AUTORIDAD}

De acuerdo al análisis de las estrategias que acompañan a las peticiones, se logran identificar y caracterizar tres formas de autoridad en las relaciones entre profesores y estudiantes: autoridad explícita, autoridad compartida y autoridad como misión moral.

\section{Forma 1. La autoridad explícita}

Esta forma de autoridad se caracteriza porque el profesor explicita constantemente las normas comportamientos y desempeños académicos que deben alcanzar los alumnos. La mayor parte de las peticiones son formuladas por el profesor (91\%) y los temas a los que se refieren son predominantemente académicos e instruccionales (78\%). En una menor 
proporción, pero igualmente significativa $(22 \%)$, las peticiones se refieren a temas 'no académicos'.

Destaca en este estilo de autoridad la estrategia para captar la atención de los estudiantes basada en permanentes preguntas que requieren de respuestas precisas que luego son validadas por el profesor. En la interacción $\mathrm{N}^{\circ} 1$ se presenta esta dinámica.

Interacción $\mathrm{N}^{\circ}$ 1. Clase de Química. GSE Alto.

Profesor: "Entonces vamos a continuar con el tema real de las cantidades químicas. Si es 1 mol de moléculas de agua, mi pregunta es ¿cuántas moléculas de agua contiene este mol? Levanten la mano.

Alumno 1: 6,02 por 10 elevado a 23

Profesor: Entonces la respuesta es: un mol de moléculas de agua contiene 6,02 por 10 elevado a 23 mol de moléculas de agua, ¿estamos claros en eso?

Alumnos: Sí

Profesor: Sí. En este caso como es un mol de molécula, este va a romper moléculas, pero también vimos el esquema de que los mol de moléculas están formadas por mol de agua y en este caso, si tenemos agua la pregunta es ¿de cuántos elementos están formados?

Alumno 2: Dos

Profesor: de dos cierto, dos de hidrógeno y uno de oxígeno. El elemento hidrógeno y el elemento oxígeno. ¿De cuántos átomos en total estaría formada una molécula?

Alumno 3: Tres

Es importante destacar en esta interacción el tipo de preguntas y la forma de presentarlas. Las preguntas son altamente estructuradas, incluso explicitadas como tal: "Mi pregunta es...". Estas solicitan una información prefijada o que se deriva de la información que recientemente ha entregado el profesor. Las respuestas son reiteradas por el mismo profesor y luego explícitamente validadas por él. Este esquema se repite permanentemente.

En esta forma de autoridad los profesores suelen utilizar las evaluaciones y calificaciones como motivos para que los estudiantes se concentren en la materia de clase. El profesor hace referencia permanentemente a evaluaciones (especialmente pruebas escritas) que aplicará próximamente. Estas referencias son altamente atendidas por los alumnos.

En la interacción $\mathrm{N}^{\circ} 2$, ocurrida al inicio de una clase, se puede apreciar cómo la profesora captura la atención de los estudiantes destacando la evaluación que tendrán ese día.

Interacción $\mathrm{N}^{\circ}$ 2. Clase de Historia. GSE Bajo.

Profesora: "Buenos días".

Alumnos: "Buenos días profesora".

Profesora: "Tomen asiento por favor." [La profesora espera que todos se sienten. Continúa parada en el centro de la sala. Mira a cada uno de los estudiantes.] "Niñitos, saquen por favor su cuaderno, y la prueba va hoy día, va al final de la clase, iya! El otro día se salvaron porque hicimos la bienvenida de la escuela, ¿Cierto?, el desayuno, la inducción, pero quedó claro que la prueba era hoy día. [Los alumnos sacan rápidamente sus cuadernos y lápices. La profesora mira a todos alumnos y espera hasta que todos estén con sus materiales en la mesa]. Bien. Chiquillos, el lunes pasado, se acuerdan, íbamos a ver de lleno la $1^{\circ}$ Guerra Mundial..." 
Es relevante en esta interacción cómo la profesora vincula el trabajo escolar a la evaluación que deberán rendir los estudiantes, la cual se presenta como un evento distinto a "la bienvenida escolar". También es relevante observar cómo la profesora se ubica en un lugar que puede ser vista por todos los estudiantes, y donde ella ve a todos. La profesora está permanentemente pendiente de crear condiciones para que su solicitud sea ejecutada por los estudiantes.

Finalmente, en este estilo es usual la justificación de la autoridad del profesor desde el rol formal y visible que asume. Los profesores recuerdan permanentemente su rol y función: "Estoy para enseñarles"; "Mi trabajo es hacerles clases". La asimetría profesor y alumnos es expuesta permanentemente; y los alumnos la reconocen. Esta situación se ejemplifica en la Interacción $\mathrm{N}^{\circ} 3$.

Interacción $\mathrm{N}^{\circ}$ 3: Clase de Historia. GSE Bajo.

[Algunos alumnos conversan] Profesora: “¡Ya!, pero antes de eso yo les voy a decir una cosa, la que echa acá soy yo, la que llama la atención soy yo, le voy a pedir a la gente que habla [rápidamente los alumnos dejan de hablar] que respondan a la clase, ¡ya!"

\section{Forma 2. La autoridad compartida}

En esta forma de autoridad el profesor comparte con sus alumnos la formulación de peticiones: $55 \%$ son expresadas por el profesor y $45 \%$ expresadas por los alumnos. El profesor dirige las actividades de enseñanza y regula las normas de comportamiento de un modo sutil, otorgando espacios para que los estudiantes intervengan activamente. Esta forma de autoridad mantiene un fuerte foco en el contenido académico: $89 \%$ de las peticiones son de tipo académico.

Destaca en este estilo las preguntas abiertas que invitan a la reflexión e imaginación. Las preguntas surgen tanto del profesor como de los estudiantes, y son acogidas e incorporadas permanentemente en la clase. En este ambiente se valora significativamente la originalidad de las preguntas y respuestas. Esta situación se puede observar claramente en la Interacción $\mathrm{N}^{\circ} 4$ y $\mathrm{N}^{\circ} 5$.

Interacción $\mathrm{N}^{\circ}$ 4: Clase de Matemática. GSE Medio.

Profesor: "Vamos a ver de dónde viene el 3,14" [El profesor da tiempo para que los estudiantes copien ejemplos de números irracionales]. "Bastián, ¿te diste cuenta por qué no pueden ser racionales?... ¿Javiera?"

Alumno 1 "Porque no se pueden transformar en fracción".

Profesor: "¿Daniela?”

Alumna 2: "Porque no se puede distinguir un período"

Profesor: "Por lo tanto, no se pueden transformar en fracción. Vamos a ver algunos irracionales destacados, por ejemplo, la longitud de la circunferencia, el diámetro de cualquier circunferencia".

Alumna 3: “¿Por qué se le puso Pi?”

Profesor: "No sé, pero lo voy a averiguar. Otro es el número de oro. ¿En qué otra situación se usa?"

Alumna 3: "Estética, arte, naturaleza" 
[El profesor muestra el símbolo de "Pi"]

Alumno 4: ¿Y qué es la "i”?

Profesor: "Lo vamos a aprender a calcular en parte de los contenidos. Ese es el objetivo de la clase de hoy, que puedan aprender a identificar los números irracionales".

[En la clase siguiente, dos días después, el Profesor retoma el tema]...

Profesor: "Carol, ¿te acuerdas que me preguntaste qué significaba Pi? A ti te gusta la lectura, yo me acordé porque sabía que era constante, pero no qué significaba. A quien le guste el arte, los colores, aquí vemos como mostraron el número y con los decimales de Pi le fueron dando forma en un caligrama. Nunca me habían preguntado eso, y lo aprendí por tu pregunta."

Interacción $\mathrm{N}^{\circ}$ 5: Clase de Física. GSE Alto

Profesora: [dirigiéndose a todos los alumnos al inicio de la clase] "Al inicio de año, hay dos reglas que yo les pedí que ustedes debían cumplir durante las clases, la postura y el silencio mientras yo esté adelante explicando. [Los alumnos dejan de hablar y atienden a la profesora. Ella prosigue] Hoy vamos a recordar que la clase anterior estuvimos definiendo lo que eran las ondas... a ver, ¿quién quiere recordarlo? [Ningún alumno responde. La profesora continúa] En la primera clase les dije que había una conducta que era necesaria para aprender física: hay que tener mucha voluntad e imaginación, porque vamos a tener que imaginar situaciones que nosotros no podemos ver, pero las podemos imaginar. Entonces, la primera pregunta que contestamos fue ¿cómo se imaginan que son las ondas?”

Es importante destacar en estas interacciones que el tipo de preguntas y exigencias se orientan a favorecer la exploración y originalidad de los estudiantes. La profesora de la Interacción $\mathrm{N}^{\circ} 5$, además de hacer una pregunta abierta, les recuerda a los alumnos las conductas que son necesarias en la clase: voluntad e imaginación. En la Interacción $\mathrm{N}^{\circ} 4$, el profesor acoge y favorece las inquietudes personales. Consistente con ello, es el referirse a los estudiantes por su nombre, poniendo énfasis en que sus intervenciones son únicas.

En esta forma de autoridad la comunicación de las normas por parte del profesor es más sutil y está centrada en el mantenimiento o continuidad del clima de trabajo. En la interacción $\mathrm{N}^{\circ} 5$ la profesora no pide explícitamente silencio; más bien recuerda una solicitud anterior. Es usual que las normas se marquen al inicio del año escolar y se espera su continuidad, que solo requiere ser recordada. Ello también se aprecia al inicio de la interacción $\mathrm{N}^{\circ} 5$.

En esta forma de autoridad el tiempo escolar aparece más prolongado. Así como la profesora de la interacción $\mathrm{N}^{\circ} 5$ refiere al inicio del año; otros profesores refieren a un avance o progreso a mediano plazo (que supera la clase actual) o, incluso a largo plazo, como son las menciones a la Prueba de Selección Universitaria (PSU) que se rinden a final de $4^{\circ}$ grado de educación secundaria (en dos años más del curso actual). Ello se presenta claramente en la Interacción $\mathrm{N}^{\circ} 6$.

Interacción $\mathrm{N}^{\circ}$ 6. Clase de Matemática. GSE Alto

Profesor: "Atienda esta materia pues este tipo de ejercicio es evaluado en la PSU y normalmente aparecen relaciones como estas: dado el triángulo, defina. Es fundamental que identifiquen los lados homólogos... Estamos bien... Cuando veamos demostraciones tendremos que justificar los criterios." 
Finalmente, cabe comentar que esta autoridad se mueve principalmente en el territorio de los contenidos de la asignatura. Asimismo, la autoridad del profesor no aparece en riesgo, más bien se asemeja a la de un capital ganado, que otorga cierta seguridad y continuidad al trabajo escolar.

\section{Forma 3. La autoridad como una misión moral}

Esta forma de autoridad se presenta exclusivamente en el GSE Bajo. Se distingue por la menor presencia de peticiones de tipo académico (55\%) y la fuerte proporción de peticiones formuladas por el profesor $(93 \%)$.

En esta forma de autoridad, el profesor se presenta como un orientador para la vida de los estudiantes. Sus peticiones se refieren a comportamientos que él estima que son "socialmente correctos", y que le significará al alumno un crecimiento personal. El profesor justifica su autoridad desde un vínculo personal orientado a 'formar para la vida'.

En esta forma, las peticiones se presentan como una ayuda a la 'toma de consciencia' y la 'personalización' de los estudiantes. En forma recurrente, los profesores solicitan a sus alumnos que cuestionen sus marcos cognitivos y valóricos, y los reemplacen por nuevos criterios para la acción. Este aspecto se manifiesta claramente en la Interacción $\mathrm{N}^{\circ} 7$.

Interacción $N^{\circ}$ 7. Clase Historia. GSE Bajo.

Profesora: [Refiriéndose a una de las campañas presidenciales de Chile de la primera mitad del siglo XX que incluyó a sectores sociales medios y bajos] "Chiquillos, ¿y Uds. están de acuerdo con estos cambios?"

Alumnos: “¡Sí!"

Profesora: “¿Por qué creen que es necesario?”

Alumno 1: "Porque o si no van seguir gobernando los de clase alta."

Profesora: "Sí, nos van a seguir gobernando la clase alta y se van a beneficiar. ¿Qué pasaría con nosotros chiquillos?"

Alumno 2: "No seríamos escuchados nosotros, no nos escucharían nuestros problemas, las necesidades que tenemos".

Profesor: "Y si la gente no conoce nuestros problemas y nuestras necesidades, ¿podrían tratar de solucionarlos?"

Alumnos [responden varios al mismo tiempo] “ $¡$ No!"

Profesor: "Hoy día chiquillos, ¿tienen los mismos derechos de una persona que nace en clase alta?"

Alumnos: «iNo!"

Profesor: "Entonces, ¿cuál sería nuestra misión el día que Uds. puedan votar? Llegar a ser presidente, esa es una opción. Lo segundo, el día en que puedan votar, es necesario votar informados de quiénes son los candidatos y cuáles son sus propuestas [Los alumnos asienten. La profesora continúa] Una pregunta, ¿Uds. solo pueden manifestarse cuándo votan?” [alumnos asienten nuevamente].

Alumna 3: [Dirigiéndose a sus compañeros de curso] "Y si no votan no tienen derecho a opinar".

Profesora: "Esa es una teoría que hoy existe de la política, pero también no votar es una forma de opinar. Eso significa el descontento. Importante chiquillos, tomemos consciencia que todos ustedes son ciudadanos y que no es necesario llegar a votar para que Uds. puedan manifestar sus ideas". 
Es relevante destacar en esta interacción cómo la profesora se presenta como parte del grupo social de sus alumnos: "Nos van a seguir gobernando"; “Qué pasaría con nosotros?” Al presentarse desde una identidad colectiva, los alumnos responden en los mismos términos: "No seríamos escuchados". Esta dinámica permite que las peticiones de la profesora (por ejemplo, la pregunta “¿cuál sería nuestra misión?”) aumenten la posibilidad de que sean acogidas pues la solicitud deja de ser una asunto meramente personal; son problemáticas que pertenecen a la comunidad.

Una imagen que podría representar esta forma de autoridad es la de una 'misión moral'. El profesor se representa como un guía que ilumina las conciencias de sus estudiantes. Aparece como cercano, transmitiendo que los problemas de sus alumnos, son también los suyos. Pese a esta cercanía, es importante advertir la fuerte asimetría que sostiene esta forma de ejercer autoridad, en cuanto el profesor actúa como un liberador social de los estudiantes.

\subsection{FORMAS DE EJERCICIO DE LA AUTORIDAD Y GSE}

Es importante advertir la dificultad de aplicar un análisis estadístico a la relación entre Autoridad Pedagógica y GSE debido al tamaño de la muestra. Sin embargo, es relevante destacar que la distribución de los profesores marca una interesante diferencia (ver Tabla 3). Como se aprecia, la forma 1 aparece con más fuerza en el GSE Bajo y la forma 2 en el GSE Alto y Medio. Si bien ello no es corroborado en el análisis estadístico, sí da cuenta de una posible diferencia que requiere ser atendida y examinada en futuros estudios.

Tabla 3. Distribución profesores en formas de autoridad

\begin{tabular}{|c|c|c|c|c|c|}
\hline & & \multicolumn{3}{|c|}{ Estilos de Autoridad } & \multirow{2}{*}{ Total } \\
\hline & & Forma 1 & Forma 2 & Forma 3 & \\
\hline \multirow{3}{*}{ GSE } & Alto & 2 & 4 & 0 & 6 \\
\hline & Medio & 2 & 4 & 0 & 6 \\
\hline & Bajo & 4 & 0 & 2 & 6 \\
\hline \multicolumn{2}{|l|}{ Total } & 8 & 8 & 2 & 18 \\
\hline
\end{tabular}

Fuente: elaboración propia.

\section{CONCLUSIONES}

El presente estudio ha logrado identificar y caracterizar tres formas de autoridad en las interacciones entre profesores y estudiantes de educación secundaria: la autoridad explícita, la autoridad compartida y la autoridad como misión moral.

En la 'autoridad explícita', el profesor asume una relación claramente directiva hacia los estudiantes, con un énfasis en el contenido académico. Destaca en esta forma el fuerte control o enmarcamiento (Bernstein, 2001) que realiza el profesor a los alumnos, ubicándolos permanentemente en el territorio de los contenidos de la asignatura. En 
esta autoridad se valora especialmente el 'hacer' del estudiante: responder las preguntas que directamente formula el profesor, cumplir con el estándar prescrito o responder a la evaluación solicitada. La autoridad se orienta hacia incorporación o mantención de los alumnos en las actividades académicas, reduce distracciones y utiliza las evaluaciones de corto plazo como una forma de control.

La 'autoridad explícita' se presenta con mayor fuerza en el GSE Bajo: cuatro de los seis profesores utilizan este estilo. Este hallazgo es consistente con los planteamientos de Delpit (1995) referido a que la autoridad es más reconocida en los sectores desaventajados cuando se presenta en forma evidente: los estudiantes valoran significativamente la pedagogía cuando es visible y cuando las solicitudes del profesor son directas. Los estudiantes del GSE Bajo de la presente investigación provienen principalmente de familias cuyos padres no sobrepasaron los 9 años de escolaridad (no terminaron la educación secundaria). Probablemente la cultura escolar se les presenta distante o inentendible en varios aspectos y materias. Ello puede explicar la alta valoración de una autoridad que explicite las reglas del juego, que precise lo que está solicitando, que indique lo que está preguntando y que evalúe lo que está enseñando; todo ello aumentará las posibilidades de que los estudiantes comprendan los códigos de esa lejana cultura, que por generaciones les ha sido ajena. Siguiendo a Delpit (1995) y Bernstein (2001), los estudiantes de sectores desaventajados tienen más posibilidades de sortear exitosamente los requerimientos de la escuela cuando el profesor hace visible las reglas del poder.

En la autoridad explícita, el profesor establece un sistema de reconocimiento y de motivación en los estudiantes que les permite incorporarlos en forma continua, capturando su atención incesantemente. Se podría interpretar que la 'autoridad explícita' actúa de tal manera como si el proceso de enseñanza y aprendizaje estuviera en permanente riesgo: en cualquier momento los alumnos podrían dejar de atender. De ahí la necesidad de movilizar permanentemente recursos personales y pedagógicos: "la que llama la atención soy yo", enfatiza una profesora a sus alumnos.

La segunda forma, denominada la 'autoridad compartida', se caracteriza en que tanto los estudiantes como el profesor formulan peticiones (45\% son de los estudiantes y $55 \%$ del profesor). El profesor dirige las actividades de enseñanza y regula las normas de comportamiento de un modo sutil, otorgando espacios para que los estudiantes intervengan activamente.

Es importante destacar que la 'autoridad compartida' se presenta en los estudiantes de mayor capital cultural: aparece en el GSE Alto y en el Medio. En estos grupos los padres tienen más de 12 años de escolaridad: han finalizado la educación secundaria y la mayor parte ha realizado estudios superiores. Probablemente, estos alumnos tienen una alta disposición hacia la enseñanza escolar, por lo cual pueden compartir el control académico con el profesor sin que ello signifique el quiebre del clima de aprendizaje.

La autoridad compartida difiere significativamente de la autoridad explícita. En esta última, se valora especialmente el 'hacer' del estudiante; en cambio, en la autoridad compartida se aprecia el 'ser' del alumno: ser creativo, original, único. Asimismo, a diferencia de la 'autoridad explícita' que se encontraba en permanente riesgo; en la 'autoridad compartida' existe un capital ganado o un piso de poder que otorga más de seguridad o certeza. En efecto, la autoridad del profesor reposa en el reconocimiento de la relevancia de lo que se está enseñando. Al respecto Zamora, Meza y Cox (2015) advierten que para los estudiantes de GSE Medio y Alto el reconocimiento de la autoridad 
se encuentra especialmente asociado a las asignaturas que tienen más horas de clases semanales y cuyos contenidos serán examinados para el ingreso a la educación superior. Dice un profesor: "atienda esta materia pues este tipo de ejercicio es evaluado en la PSU" (Prueba de Selección Universitaria). Se podría interpretar que en la 'autoridad compartida' el profesor aparece con un modo sutil (otorgando más espacios para que los estudiantes intervengan) debido a que su autoridad descansa no solo en sus capacidades, sino también en la aceptación previa de que está en juego algo relevante: la energía del profesor no es tanto incorporar a los estudiantes, como el de mantener un clima de trabajo ya logrado para que ellos puedan progresar en el aprendizaje y desarrollar sus cualidades personales.

La tercera forma de autoridad, como 'una misión moral', se presenta exclusivamente en el GSE Bajo. Esta se distingue por la menor presencia de contenidos académicos y la alta directividad del profesor. Las interacciones se orientan a cuestionar los marcos cognitivos y valóricos de los estudiantes, y a influir para que adhieran a nuevos principios de acción. Es característica de esta forma la utilización de los contenidos de la asignatura para interpelar los comportamientos y decisiones de los estudiantes. De este modo, el contenido se transforma en una directriz moral.

Esta forma de autoridad recuerda al 'sacerdote laico' descrito por Durkheim (2009), donde el profesor es el mandatario de la moral social y encarna los valores universales. Ciertamente, el profesor se presenta con una fuerte convicción moral, proyectándose como un orientador de la vida de sus estudiantes. Sin embargo, esta autoridad tiene su propia particularidad: la cercanía con los alumnos. Los profesores se vinculan personalmente con los estudiantes, valorando sus problemáticas como si fuesen de los propios maestros. Por tanto, la autoridad, además de promover una adhesión moral, se orienta a relevar una identidad comunitaria. Aquí el profesor aparece como el más genuino representante de la comunidad: él puede hablar a la comunidad de "nuestros problemas" y él puede preguntar acerca de "nuestra misión". Mientras que para Durkheim (2009) el maestro es el intérprete de los valores sociales que están por sobre él y por sobre sus alumnos; en esta autoridad, el profesor se presenta como el intérprete de la valores y anhelos de la propia comunidad. De este modo, la presente autoridad se asemeja a un líder moral que tiene la lucidez para expresar lo que su comunidad necesita.

Considerando esta dinámica, es coherente que la autoridad como misión moral se presente en el GSE Bajo. Es aquí donde la identidad social adquiere preeminencia por sobre la búsqueda de una identidad individualizada (Bernstein, 1989). La voz comunitaria hace que tengan más fuerzas las reivindicaciones de los sectores sociales más postergados, como también permite que se puedan sobrellevar los dolores acumulados.

Finalmente, es relevante comentar que la autoridad como 'misión moral' no es la única posibilidad para GSE Bajo. Es importante destacar la presencia de la 'autoridad explícita', la cual está más centrada en los contenidos académicos. Por consiguiente, se puede señalar que la autoridad del profesor en los sectores desaventajados se debate hoy entre constituirse en un líder moral u ofrecer una pedagogía visible centrada en los contenidos académicos.

\section{REFERENCIAS BIBLIOGRÁFICAS}

Arendt, H. (1996). Entre el pasado y el futuro. Barcelona: Península.

Batallán, G. (2003). El poder y la autoridad en la escuela. La conflictividad de las relaciones escolares 
desde la perspectiva de los docentes de infancia. Revista Mexicana de Investigación Educativa, 8(19), 679-704.

Bernstein, B. (1989). Clases, códigos y control. Madrid: Akal.

Bernstein, B. (2001). La estructura del discurso pedagógico. Madrid: Morata.

Bourdieu, P., \& Passeron, J. (1996). La reproducción: elementos para una teoría del sistema de enseñanza. Ciudad de México: Laia.

Centro de Investigación y Desarrollo de la Educación. (2012). IX Encuesta Nacional, Actores del sistema educativo. Santiago de Chile: CIDE, Universidad Alberto Hurtado.

Delpil, L. (1995). Others People's Children. Cultural conflict in the classroom. New York: New Press.

Dubet, F. (2006). El declive de la institución. Barcelona: Gedisa.

Dubet, F., \& Martuccelli, D. (1998). En la escuela: sociología de la experiencia escolar. Buenos Aires: Losada.

Durkheim, E. (2009). Educación y Socialización. Buenos Aires: Proa.

Harjünen, E. (2009). How do teachers view their own pedagogical authority? Teachers and Teaching: Theory and Practice, 15(1), 87-107. doi:10.1080 /13540600802661345

Martuccelli, M. (2009). La autoridad en las salas de clase. Problemas estructurales y márgenes de acción. Diversia, 1, 99-128.

Meza, M., Cox, P., \& Zamora, G. (2015). ¿Qué y cómo observar interacciones para comprender la autoridad pedagógica del profesor en su ejercicio? Educação e Pesquisa, 41(3), 729-742.

PISA. (2009). Programa Internacional de Evaluación de Estudiantes. Santiago: Ministerio de Educación de Chile.

Ruffinelli, A. (2014) Dificultades de la iniciación docente: ¿iguales para todos? Estudios Pedagógicos, $X L(1), 229-242$.

Tedesco, J.C., \& Tenti, E. (2002). Nuevos tiempos y nuevos docentes. Brasilia: Ministerio de Educação.

Weber, M. (1983). Economía y sociedad: esbozo de sociología comprensiva. México: FCE.

Zamora, G., Meza, M., \& Cox, P. (2015). ¿De dónde surge la autoridad de los profesores chilenos? Análisis desde las perspectivas de los estudiantes. MAGIS, Revista Internacional de Investigación en Educación, 7(15), 63-80. doi:10.1590/s1517-97022015051777

Zerón, A. (2006). Sentido de la violencia escolar. Un estudio de sociología comprensiva. Santiago de Chile (Tesis Doctorado en Educación). Pontificia Universidad Católica de Chile. 
